\title{
On Fixed Point Results in Controlled Metric Spaces
}

\author{
Jamshaid Ahmad $\mathbb{D},{ }^{1}$ Abdullah Eqal Al-Mazrooei, ${ }^{1}$ Hassen Aydi $\mathbb{D},{ }^{2,3}$ \\ and Manuel De la Sen $\left.{ }^{4}\right)^{4}$ \\ ${ }^{1}$ Department of Mathematics, University of Jeddah, P.O. Box 80327, Jeddah 21589, Saudi Arabia \\ ${ }^{2}$ University of Sousse, Higher Institute of Information Technology and Communication Techniques, H. Sousse 4000, Tunisia \\ ${ }^{3}$ China Medical University Hospital, China Medical University, Taichung 40402, Taiwan \\ ${ }^{4}$ Institute of Research and Development of Processes IIDP, University of the Basque Country, Campus of Leioa. Leioa (Bizkaia), \\ P.O. Box 48940, Spain
}

Correspondence should be addressed to Hassen Aydi; hassen.aydi@isima.rnu.tn

Received 7 June 2020; Revised 22 August 2020; Accepted 5 September 2020; Published 24 November 2020

Academic Editor: Adrian Petrusel

Copyright (c) 2020 Jamshaid Ahmad et al. This is an open access article distributed under the Creative Commons Attribution License, which permits unrestricted use, distribution, and reproduction in any medium, provided the original work is properly cited.

\begin{abstract}
In this article, we introduce Reich type contractions and $(\alpha, F)$-contractions in the class of controlled metric spaces and establish some new related fixed point theorems. Our results are generalizations of some known results of literature. Some examples and certain consequences are given to illustrate significance of presented results.
\end{abstract}

\section{Introduction and Preliminaries}

In 1993, Czerwik [1] reintroduced a new class of generalized metric spaces, called as $b$-metric spaces, as generalizations of metric spaces.

Definition 1 ([1]). Let $Y$ be a nonempty set and $s \geq 1$. A function $d_{b}: Y \times Y \longrightarrow[0, \infty)$ is said to be a $b$-metric if for all $\varsigma, \omega, \tau \in Y$,

(b1) $d_{b}(\varsigma, \omega)=0$ iff $\varsigma=\omega$

(b2) $d_{b}(\varsigma, \omega)=d_{b}(\omega, \varsigma)$ for all $\varsigma, \omega \in Y$

(b3) $d_{b}(\varsigma, \tau) \leq s\left[d_{b}(\varsigma, \omega)+d_{b}(\omega, \tau)\right]$

The pair $\left(Y, d_{b}\right)$ is then called a $b$-metric space. Subsequently, many fixed point results on such spaces were given (see [2-7]).

Kamran et al. [8] initiated the concept of extended $b$ -metric spaces.

Definition 2. Let $Y$ be a nonempty set and $p: Y \times Y \longrightarrow$ $[1, \infty)$ be a function. A function $d_{e}: Y \times Y \longrightarrow[0, \infty)$ is called an extended $b$-metric if for all $\varsigma, \omega, \tau \in Y$, (i) $d_{e}(\varsigma, \omega)=0$ iff $\varsigma=\omega$

(ii) $d_{e}(\varsigma, \omega)=d_{e}(\omega, \varsigma)$

(iii) $d_{e}(\varsigma, \omega) \leq p(\varsigma, \omega)\left[d_{e}(\varsigma, \tau)+d_{e}(\tau, \omega)\right]$

The pair $\left(Y, d_{e}\right)$ is called an extended $b$-metric space.

Very recently, a new kind of a generalized $b$-metric space was introduced by Mlaiki et al. [9].

Definition 3. Let $Y$ be a nonempty set and $p: Y \times Y \longrightarrow$ $[1, \infty)$ be a function. A function $\mathscr{C}: Y \times Y \longrightarrow[0, \infty)$ is called a controlled metric if for all $\varsigma, \omega, \tau \in Y$,

(i) $\mathscr{C}(\varsigma, \omega)=0$ iff $\varsigma=\omega$

(ii) $\mathscr{C}(\varsigma, \omega)=C(\omega, \varsigma)$

(iii) $\mathscr{C}(\varsigma, \omega) \leq p(\varsigma, \tau) \mathscr{C}(\varsigma, \tau)+p(\omega, \tau) \mathscr{C}(\omega, \tau)$

The pair $(Y, \mathscr{C})$ is called a controlled metric space (see also [10]).

The Cauchy and convergent sequences in controlled metric type spaces are defined in this way. 
Definition 4 ([9]). Let $(Y, \mathscr{C})$ be a controlled metric space and $\left\{\varsigma_{n}\right\}_{n \geq 0}$ be a sequence in $Y$. Then,

(i) The sequence $\left\{\varsigma_{n}\right\}$ converges to some $\varsigma$ in $Y$; if for every $\varepsilon>0$, there exists $N=N(\varepsilon) \in \mathbb{N}$ such that $\mathscr{C}\left(\varsigma_{n}, \varsigma\right)<\varepsilon$ for all $n \geq N$. In this case, we write $\lim _{n \rightarrow \infty} \varsigma_{n}=\varsigma$

(ii) The sequence $\left\{\varsigma_{n}\right\}$ is Cauchy; if for every $\varepsilon>0$, there exists $N=N(\varepsilon) \in \mathbb{N}$ such that $\mathscr{C}\left(\varsigma_{m}, \varsigma_{n}\right)<\varepsilon$ for all $m, n \geq N$

(iii) The controlled metric space $(Y, \mathscr{C})$ is called complete if every Cauchy sequence is convergent

Definition 5 ([9]). Let $(Y, \mathscr{C})$ be a controlled metric space. Let $\varsigma \in Y$ and $\varepsilon>0$.

(i) The open ball $B(\varsigma, \varepsilon)$ is

$$
B(\varsigma, \varepsilon)=\{\omega \in Y: \mathscr{C}(\varsigma, \omega) \triangleleft \mathcal{E}\}
$$

(ii) The mapping $\Omega: Y \longrightarrow Y$ is said to be continuous at $\varsigma \in Y$; if for all $\varepsilon>0$, there exists $\delta>0$ such that $\Omega(B(\varsigma, \delta)) \subseteq B(\Omega \varsigma, \varepsilon)$

Very recently, Wardowski [11] introduced a new type of contractions, called $F$-contractions and established some new related fixed point theorems in the context of complete metric spaces.

Definition 6. Let $F: \mathbb{R}^{+} \longrightarrow \mathbb{R}$ be a function satisfying

$\left(F_{1}\right) F$ is strictly increasing, that is, for all $t_{1}, t_{2} \in \mathbb{R}^{+}$such that $t_{1}<t_{2}$ implies that $F\left(t_{1}\right)<F\left(t_{2}\right)$.

$\left(F_{2}\right)$ For every sequence $\left\{t_{n}\right\}$ of positive real numbers, $\lim _{n \rightarrow \infty} t_{n}=0$ and $\lim _{n \rightarrow \infty} F\left(t_{n}\right)=-\infty$ are equivalent.

$\left(F_{3}\right)$ There is $h \in(0,1)$ so that $\lim _{t \rightarrow 0^{+}} t^{h} F(t)=0$.

Let $\sqsubseteq$ be the set of above functions $F$ satisfying $\left(F_{1}\right)-\left(F_{3}\right)$ (to be consistent with Wardowski [11]). A self-mapping $\Omega$ on the metric space $(Y, \eta)$ is said to be an $F$-contraction if there are a function $F$ satisfying $\left(F_{1}\right)-\left(F_{3}\right)$ and a constant $\tau>0$ so that

$$
\eta(\Omega \varsigma, \Omega \omega)>0 \Rightarrow \tau+F(\eta(\Omega \varsigma, \Omega \omega)) \leq F(\eta(\varsigma, \omega))
$$

for all $\varsigma, \omega \in Y$.

Theorem 7 [11]. Let $(Y, \eta)$ be a complete metric space and $\Omega: Y \longrightarrow Y$ be an $F$-contraction, then $\Omega$ admits a unique fixed point.

The authors in [11] manifested that a Banach contraction is a specific case of F-contractions, while there are many $F$ -contractions which need not be a Banach contraction. For more details, we refer the readers to ([12-22]).

In this paper, we first define Reich [23, 24] and $(\alpha, F)$-contractions in the setting of controlled metric spaces and prove some new fixed point results. We also provide some examples to illustrate significance of the established results.

\section{Results on Reich Type Contractions}

Theorem 8. Let $(Y, \mathscr{C})$ be a complete controlled metric space. Let $\Omega: Y \longrightarrow Y$ be so that there are $\alpha, \beta, \gamma \in(0,1)$ with $k=$ $\alpha+\beta / 1-\gamma<1$,

$$
\mathscr{C}(\Omega \varsigma, \Omega \omega) \leq \alpha \mathscr{C}(\varsigma, \omega)+\beta \mathscr{C}(\varsigma, \Omega \varsigma)+\gamma \mathscr{C}(\omega, \Omega \omega)
$$

for all $\varsigma, \omega \in Y$. For $\varsigma_{0} \in Y$, take $\varsigma_{n}=\Omega^{n} \varsigma_{0}$. Assume that

$$
\sup _{m \geq 1} \lim _{i \rightarrow \infty} \frac{p\left(\varsigma_{i+1}, \varsigma_{i+2}\right) p\left(\varsigma_{i+1}, \varsigma_{m}\right)}{p\left(\varsigma_{i}, \varsigma_{i+1}\right)}<\frac{1}{k} .
$$

Suppose that $\lim _{n \rightarrow \infty} p\left(\varsigma_{n}, \varsigma\right)$ and $\lim _{n \rightarrow \infty} p\left(\varsigma, \varsigma_{n}\right)$ exist, are finite, and $\gamma \lim _{n \rightarrow \infty} p\left(\varsigma_{n}, \varsigma\right)<1$ for every $\varsigma \in Y$, then $\Omega$ possesses a unique fixed point.

Proof. The considered sequence $\left\{\varsigma_{n}\right\}$ verifies $\varsigma_{n+1}=\Omega \varsigma_{n}$ for all $n \in \mathbb{N}$. Obviously, if there exists $n_{0} \in \mathbb{N}$ for which $\varsigma_{n_{0}+1}=$ $\varsigma_{n_{0}}$, then $\Omega \varsigma_{n_{0}}=\varsigma_{n_{0}}$, and the proof is finished. Thus, we suppose that $\varsigma_{n+1}=\varsigma_{n}$ for every $n \in \mathbb{N}$. Thus, by (1), we have

$$
\begin{aligned}
\mathscr{C}\left(\varsigma_{n}, \varsigma_{n+1}\right)= & \mathscr{C}\left(\Omega \varsigma_{n-1}, \Omega \varsigma_{n}\right) \leq \alpha \mathscr{C}\left(\varsigma_{n-1}, \varsigma_{n}\right) \\
& +\beta \mathscr{C}\left(\varsigma_{n-1}, \Omega \varsigma_{n-1}\right)+\gamma \mathscr{C}\left(\varsigma_{n}, \Omega \varsigma_{n}\right) \\
= & \alpha \mathscr{C}\left(\varsigma_{n-1}, \varsigma_{n}\right)+\beta \mathscr{C}\left(\varsigma_{n-1}, \varsigma_{n}\right)+\gamma \mathscr{C}\left(\varsigma_{n}, \varsigma_{n+1}\right)
\end{aligned}
$$

which implies that

$$
\mathscr{C}\left(\varsigma_{n}, \varsigma_{n+1}\right) \leq \frac{\alpha+\beta}{1-\gamma} \mathscr{C}\left(\varsigma_{n-1}, \varsigma_{n}\right)=k \mathscr{C}\left(\varsigma_{n-1}, \varsigma_{n}\right) .
$$

Thus, we have

$\mathscr{C}\left(\varsigma_{n}, \varsigma_{n+1}\right) \leq k \mathscr{C}\left(\varsigma_{n-1}, \varsigma_{n}\right) \leq k^{2} \mathscr{C}\left(\varsigma_{n-2}, \varsigma_{n-1}\right) \leq \cdots \leq k^{n} \mathscr{C}\left(\varsigma_{0}, \varsigma_{1}\right)$.

For all $n, m \in \mathbb{N}(n<m)$, we have

$$
\begin{aligned}
\mathscr{C}\left(\varsigma_{n}, \varsigma_{m}\right) \leq & p\left(\varsigma_{n}, \varsigma_{n+1}\right) \mathscr{C}\left(\varsigma_{n}, \varsigma_{n+1}\right)+p\left(\varsigma_{n+1}, \varsigma_{m}\right) \mathscr{C}\left(\varsigma_{n+1}, \varsigma_{m}\right) \\
\leq & p\left(\varsigma_{n}, \varsigma_{n+1}\right) \mathscr{C}\left(\varsigma_{n}, \varsigma_{n+1}\right)+p\left(\varsigma_{n+1}, \varsigma_{m}\right) p\left(\varsigma_{n+1}, \varsigma_{n+2}\right) \\
& \cdot \mathscr{C}\left(\varsigma_{n+1}, \varsigma_{n+2}\right)+p\left(\varsigma_{n+1}, \varsigma_{m}\right) p\left(\varsigma_{n+2}, \varsigma_{m}\right) \mathscr{C}\left(\varsigma_{n+2}, \varsigma_{m}\right) \\
\leq & p\left(\varsigma_{n}, \varsigma_{n+1}\right) \mathscr{C}\left(\varsigma_{n}, \varsigma_{n+1}\right)+p\left(\varsigma_{n+1}, \varsigma_{m}\right) p\left(\varsigma_{n+1}, \varsigma_{n+2}\right) \\
& \cdot \mathscr{C}\left(\varsigma_{n+1}, \varsigma_{n+2}\right)+p\left(\varsigma_{n+1}, \varsigma_{m}\right) p\left(\varsigma_{n+2}, \varsigma_{m}\right) p\left(\varsigma_{n+2}, \varsigma_{n+3}\right) \\
& \cdot \mathscr{C}\left(\varsigma_{n+2}, \varsigma_{n+3}\right)+p\left(\varsigma_{n+1}, \varsigma_{m}\right) p\left(\varsigma_{n+2}, \varsigma_{m}\right) p\left(\varsigma_{n+3}, \varsigma_{m}\right) \\
& \cdot \mathscr{C}\left(\varsigma_{n+3}, \varsigma_{m}\right) \leq \cdots \leq p\left(\varsigma_{n}, \varsigma_{n+1}\right) \mathscr{C}\left(\varsigma_{n}, \varsigma_{n+1}\right) \\
& +\sum_{i=n+1}^{m-2}\left(\prod_{j=n+1}^{i} p\left(\varsigma_{j}, \varsigma_{m}\right)\right) p\left(\varsigma_{i}, \varsigma_{i+1}\right) \mathscr{C}\left(\varsigma_{i}, \varsigma_{i+1}\right) \\
& +\prod_{i=n+1}^{m-1} p\left(\varsigma_{i}, \varsigma_{m}\right) \mathscr{C}\left(\varsigma_{m-1}, \varsigma_{m}\right) .
\end{aligned}
$$


This implies that

$$
\begin{aligned}
\mathscr{C}\left(\varsigma_{n}, \varsigma_{m}\right) \leq & p\left(\varsigma_{n}, \varsigma_{n+1}\right) \mathscr{C}\left(\varsigma_{n}, \varsigma_{n+1}\right) \\
& +\sum_{i=n+1}^{m-2}\left(\prod_{j=n+1}^{i} p\left(\varsigma_{j}, \varsigma_{m}\right)\right) p\left(\varsigma_{i}, \varsigma_{i+1}\right) \mathscr{C}\left(\varsigma_{i}, \varsigma_{i+1}\right) \\
& +\left(\prod_{i=n+1}^{m-1} p\left(\varsigma_{i}, \varsigma_{m}\right)\right) p\left(\varsigma_{m-1}, \varsigma_{m}\right) \mathscr{C}\left(\varsigma_{m-1}, \varsigma_{m}\right) \\
\leq & p\left(\varsigma_{n}, \varsigma_{n+1}\right) k^{n} \mathscr{C}\left(\varsigma_{0}, \varsigma_{1}\right) \\
& +\sum_{i=n+1}^{m-2}\left(\prod_{j=n+1}^{i} p\left(\varsigma_{j}, \varsigma_{m}\right)\right) p\left(\varsigma_{i}, \varsigma_{i+1}\right) k^{i} \mathscr{C}\left(\varsigma_{0}, \varsigma_{1}\right) \\
& +\left(\prod_{i=n+1}^{m-1} p\left(\varsigma_{i}, \varsigma_{m}\right)\right) p\left(\varsigma_{m-1}, \varsigma_{m}\right) k^{m-1} \mathscr{C}\left(\varsigma_{0}, \varsigma_{1}\right) \\
= & p\left(\varsigma_{n}, \varsigma_{n+1}\right) k^{n} \mathscr{C}\left(\varsigma_{0}, \varsigma_{1}\right) \\
& +\sum_{i=n+1}^{m-1}\left(\prod_{j=n+1}^{i} p\left(\varsigma_{j}, \varsigma_{m}\right)\right) p\left(\varsigma_{i}, \varsigma_{i+1}\right) k^{i} \mathscr{C}\left(\varsigma_{0}, \varsigma_{1}\right) .
\end{aligned}
$$

Let

$$
S_{l}=\sum_{i=0}^{l}\left(\prod_{j=0}^{i} p\left(\varsigma_{j}, \varsigma_{m}\right)\right) p\left(\varsigma_{i}, \varsigma_{i+1}\right) k^{i} \mathscr{C}\left(\varsigma_{0}, \varsigma_{1}\right) .
$$

Consider

$$
v_{i}=\left(\prod_{j=0}^{i} p\left(\varsigma_{j}, \varsigma_{m}\right)\right) p\left(\varsigma_{i}, \varsigma_{i+1}\right) k^{i} \mathscr{C}\left(\varsigma_{0}, \varsigma_{1}\right) .
$$

We have

$$
\frac{v_{i+1}}{v_{i}}=p\left(\varsigma_{i+1}, \varsigma_{m}\right) \frac{p\left(\varsigma_{i+1}, \varsigma_{i+2}\right)}{p\left(\varsigma_{i}, \varsigma_{i+1}\right)} k .
$$

In view of condition (4) and the ratio test, we ensure that the series $\sum_{i} v_{i}$ converges. Thus, $\lim _{n \rightarrow \infty} S_{n}$ exists. Hence, the real sequence $\left\{S_{n}\right\}$ is Cauchy.

Now, using (9), we get

$$
\mathscr{C}\left(\varsigma_{n}, \varsigma_{m}\right) \leq \mathscr{C}\left(\varsigma_{0}, \varsigma_{1}\right)\left[k^{n} p\left(\varsigma_{n}, \varsigma_{n+1}\right)+\left(S_{m-1}-S_{n}\right)\right]
$$

Above, we used $p(\varsigma, \omega) \geq 1$. Letting $n, m \longrightarrow \infty$ in (13), we obtain

$$
\lim _{n, m \rightarrow \infty} \mathscr{C}\left(\varsigma_{n}, \varsigma_{m}\right)=0
$$

Thus, the sequence $\left\{\varsigma_{n}\right\}$ is Cauchy in the complete controlled metric space $(Y, \mathscr{C})$. So, there is some $\varsigma^{*} \in Y$ so that

$$
\lim _{n \rightarrow \infty} \mathscr{C}\left(\varsigma_{n}, \varsigma^{*}\right)=0
$$

that is, $\varsigma_{n} \longrightarrow \varsigma^{*}$ as $n \longrightarrow \infty$. Now, we will prove that $\varsigma^{*}$ is a fixed point of $\Omega$. By (3) and condition (iii), we get

$$
\begin{aligned}
\mathscr{C}\left(\varsigma^{*}, \Omega \varsigma^{*}\right) \leq & p\left(\varsigma^{*}, \varsigma_{n+1}\right) \mathscr{C}\left(\varsigma^{*}, \varsigma_{n+1}\right)+p\left(\varsigma_{n+1}, \Omega \varsigma^{*}\right) \mathscr{C}\left(\varsigma_{n+1}, \Omega \varsigma^{*}\right) \\
= & p\left(\varsigma^{*}, \varsigma_{n+1}\right) \mathscr{C}\left(\varsigma^{*}, \varsigma_{n+1}\right)+p\left(\varsigma_{n+1}, \Omega \varsigma^{*}\right) \mathscr{C}\left(\Omega \varsigma_{n}, \Omega \varsigma^{*}\right) \\
\leq & p\left(\varsigma^{*}, \varsigma_{n+1}\right) \mathscr{C}\left(\varsigma^{*}, \varsigma_{n+1}\right)+p\left(\varsigma_{n+1}, \Omega \varsigma^{*}\right)\left[\alpha \mathscr{C}\left(\varsigma_{n}, \varsigma^{*}\right)\right. \\
& \left.+\beta \mathscr{C}\left(\varsigma_{n}, \Omega \varsigma_{n}\right)+\gamma \mathscr{C}\left(\varsigma^{*}, \Omega \varsigma^{*}\right)\right] \\
= & p\left(\varsigma^{*}, \varsigma_{n+1}\right) \mathscr{C}\left(\varsigma^{*}, \varsigma_{n+1}\right)+p\left(\varsigma_{n+1}, \Omega \varsigma^{*}\right)\left[\alpha \mathscr{C}\left(\varsigma_{n}, \varsigma^{*}\right)\right. \\
& \left.+\beta \mathscr{C}\left(\varsigma_{n}, \varsigma_{n+1}\right)+\gamma \mathscr{C}\left(\varsigma^{*}, \Omega \varsigma^{*}\right)\right] .
\end{aligned}
$$

Taking the limit as $n \longrightarrow \infty$ and using $(5,6)$ and the fact that $\lim _{n \rightarrow \infty} p\left(\varsigma_{n}, \varsigma\right)$ and $\lim _{n \rightarrow \infty} p\left(\varsigma, \varsigma_{n}\right)$ exist, are finite, we obtain that

$$
\mathscr{C}\left(\varsigma^{*}, \Omega \varsigma^{*}\right) \leq\left[\gamma \lim _{n \rightarrow \infty} p\left(\varsigma_{n+1}, \Omega \varsigma^{*}\right)\right] \mathscr{C}\left(\varsigma^{*}, \Omega \varsigma^{*}\right)
$$

Suppose that $\varsigma^{*} \neq \Omega \varsigma^{*}$, having in mind that $\left[\gamma \lim _{n \rightarrow \infty}\right.$ $\left.p\left(\varsigma_{n+1}, \Omega \varsigma^{*}\right)\right]<1$, so

$$
0<\mathscr{C}\left(\varsigma^{*}, \Omega \varsigma^{*}\right) \leq\left[\gamma \lim _{n \rightarrow \infty} p\left(\varsigma_{n+1}, \Omega \varsigma^{*}\right)\right] \mathscr{C}\left(\varsigma^{*}, \Omega \varsigma^{*}\right)<\mathscr{C}\left(\varsigma^{*}, \Omega \varsigma^{*}\right) .
$$

It is a contradiction. This yields that $\varsigma^{*}=\Omega \varsigma^{*}$. The uniqueness of the fixed point follows easily. It completes the proof.

Example 9. Consider $Y=\{0,1,2\}$. Take the controlled metric $\mathscr{C}$ defined as

$$
\mathscr{C}(0,1)=\frac{1}{2}, \mathscr{C}(0,2)=\frac{11}{20}, \mathscr{C}(1,2)=\frac{3}{20},
$$

where $p: Y \times Y \longrightarrow[1, \infty)$ is symmetric such that

$p(0,0)=p(1,1)=p(2,2)=p(1,2)=1, p(0,2)=2, p(0,1)=\frac{3}{2}$.

Given $\Omega: Y \longrightarrow Y$ as

$$
\Omega 0=2 \text { and } \Omega 1=\Omega 2=1 .
$$

Consider $\alpha=1 / 11$ and $\beta=\gamma=3 / 11$. Take $\varsigma_{0}=0$, then $\varsigma_{1}=2$ and $\varsigma_{n}=1$ for all $n \geq 2$. Clearly, (4) is satisfied. On the other hand, note that (3) holds for all $\varsigma, \omega \in Y$. All other hypotheses of Theorem 8 are verified, and so $\Omega$ has a unique fixed point, which is $u=1$.

Example 10. Let $Y=[0,1]$. Consider the controlled metric type $\mathscr{C}$ defined as

$$
\mathscr{C}(\varsigma, \omega)=|\varsigma-\omega|^{2}
$$

where $p(\varsigma, \omega)=\varsigma+\omega+1$ for $\varsigma, \omega \in Y$. Take $\Omega \varsigma=\varsigma^{2} / 4$. Consider $\alpha=1 / 4$ and $\beta=\gamma=1 / 3$. Take $\varsigma_{0}=0$; so, (4) is satisfied. 
Also, (3) holds. All conditions in Theorem 8 are fulfilled, and so, there is a unique fixed point, which is $u=0$.

Corollary 11 (see. [9]). Let $(Y, \mathscr{C})$ be a complete controlled metric space. Let $\Omega: Y \longrightarrow Y$ be s that there are $\alpha \in(0,1)$ and

$$
\mathscr{C}(\Omega \varsigma, \Omega \omega) \leq \alpha \mathscr{C}(\varsigma, \omega)
$$

for all $\varsigma, \omega \in Y$. For $\varsigma_{0} \in Y$, take $\varsigma_{n}=\Omega^{n} \varsigma_{0}$. Assume that $\sup _{m \geq 1} \lim _{i \rightarrow \infty} p\left(\varsigma_{i+1}, \varsigma_{i+2}\right) p\left(\varsigma_{i+1}, \varsigma_{m}\right) / p\left(\varsigma_{i}, \varsigma_{i+1}\right)<1 / \alpha .(36)$

Suppose that $\lim _{n \rightarrow \infty} p\left(\varsigma_{n}, \varsigma\right)$ and $\lim _{n \rightarrow \infty} p\left(\varsigma, \varsigma_{n}\right)$ exist, are finite, and $\gamma \lim _{n \rightarrow \infty} p\left(\varsigma_{n}, \varsigma\right)<1$ for every $\varsigma \in Y$, then $\Omega$ possesses a unique fixed point.

Proof. Taking $\beta=\gamma=0$ in Theorem 8 .

\section{Results on $(\alpha, F)$-Contractions}

In 2012, Samet et al. [25] initiated the notion of $\alpha$-admissible mappings and proved some related fixed point results in the context of complete metric spaces.

Definition 12 ([25]). Let $Y$ be a nonempty set, and $\alpha: Y \times Y$ $\longrightarrow[0, \infty)$ be a given function. A self-mapping $\Omega$ on $Y$ is called $\alpha$-admissible if

$$
\varsigma, \omega \in Y, \alpha(\varsigma, \omega) \geq 1 \Rightarrow \alpha(\Omega \varsigma, \Omega \omega) \geq 1 \text {. }
$$

Definition 13. Let $(Y, \mathscr{C})$ be a controlled metric space. A mapping $\Omega: Y \longrightarrow Y$ is said to be an $(\alpha, F)$-contraction if there are some $\alpha: Y \times Y \longrightarrow \mathbb{R}^{+}, F \in \sqsubseteq$, and $\tau>0$ so that

$$
\tau+\alpha(\varsigma, \omega) F((\mathscr{C}(\Omega \varsigma, \Omega \omega)) \leq F(\mathscr{C}(\varsigma, \omega))
$$

for all $\varsigma, \omega \in Y$ with $\mathscr{C}(\Omega \varsigma, \Omega \omega)>0$.

Theorem 14. Let $(Y, \mathscr{C})$ be a complete controlled metric space. Let $\Omega: Y \longrightarrow Y$ be an $(\alpha, F)$-contraction so that

(i) $\Omega$ is $\alpha$-admissible

(ii) There is $\varsigma_{0} \in Y$ so that $\alpha\left(\varsigma_{0}, \Omega \varsigma_{0}\right) \geq 1$

(iii) $\Omega$ is continuous

(iv) For $\varsigma_{0} \in Y$, define the Picard sequence $\left\{\varsigma_{n}=\Omega^{n} \varsigma_{0}\right\}$ such that

$$
\sup _{m \geq 1} \lim _{i \rightarrow \infty} \frac{p\left(\varsigma_{i+1}, \varsigma_{i+2}\right) p\left(\varsigma_{i+1}, \varsigma_{m}\right)}{p\left(\varsigma_{i}, \varsigma_{i+1}\right)}<1 .
$$

Assume that $\lim _{n \rightarrow \infty} p\left(\varsigma_{n}, \varsigma\right)$ and $\lim _{n \rightarrow \infty} p\left(\varsigma, \varsigma_{n}\right)$ exist and are finite, for every $\varsigma \in Y$, then $\Omega$ possesses a unique fixed point.

Proof. Let $\varsigma_{0} \in Y$ be such that $\alpha\left(\varsigma_{0}, \Omega \varsigma_{0}\right) \geq 1$. We define a sequence $\left\{\varsigma_{n}\right\}$ in $Y$ by $\varsigma_{n+1}=\Omega \varsigma_{n}$ for all $n \in \mathbb{N}$. Clearly, if there is $n_{0}$ so that $\varsigma_{n_{0}+1}=\varsigma_{n_{0}}$, then the proof is finished. So, assume that $\varsigma_{n+1}=\varsigma_{n}$ for each $n \in \mathbb{N}$, by using (i) and (ii), it is obvious that

$$
\alpha\left(\varsigma_{n}, \varsigma_{n+1}\right) \geq 1
$$

for all $n \in \mathbb{N}$. By (25), we have

$$
\begin{aligned}
\tau+F\left(\mathscr{C}\left(\varsigma_{n}, \varsigma_{n+1}\right)\right)= & +F\left(\mathscr{C}\left(\Omega \varsigma_{n-1}, \Omega \varsigma_{n}\right)\right) \leq \tau \\
& +\alpha\left(\varsigma_{n}, \varsigma_{n+1}\right) F\left(\mathscr{C}\left(\Omega \varsigma_{n-1}, \Omega \varsigma_{n}\right)\right) .
\end{aligned}
$$

Since $\Omega$ is an $(\alpha, F)$-contraction, we can write

$$
\begin{aligned}
\tau+F\left(\mathscr{C}\left(\varsigma_{n}, \varsigma_{n+1}\right)\right) & \leq \tau+\alpha\left(\varsigma_{n}, \varsigma_{n+1}\right) F\left(\mathscr{C}\left(\Omega \varsigma_{n-1}, \Omega \varsigma_{n}\right)\right) \\
& \leq F\left(\mathscr{C}\left(\varsigma_{n-1}, \varsigma_{n}\right)\right) .
\end{aligned}
$$

Thus, from (29), we get

$$
\begin{aligned}
F\left(\mathscr{C}\left(\varsigma_{n}, \varsigma_{n+1}\right)\right) & \leq F\left(\mathscr{C}\left(\varsigma_{n-1}, \varsigma_{n}\right)\right)-\tau \leq F\left(\mathscr{C}\left(\varsigma_{n-2}, \varsigma_{n-1}\right)\right)-2 \tau \\
& \leq F\left(\mathscr{C}\left(\varsigma_{n-3}, \varsigma_{n-2}\right)\right)-3 \tau \leq \cdots \leq F\left(\mathscr{C}\left(\varsigma_{0}, \varsigma_{1}\right)\right) .-n \tau
\end{aligned}
$$

Thus, by (29), we have

$$
F\left(\mathscr{C}\left(\varsigma_{n}, \varsigma_{n+1}\right)\right) \leq \leq F\left(\mathscr{C}\left(\varsigma_{0}, \varsigma_{1}\right)\right)-n \tau
$$

Letting $n \longrightarrow \infty$ in (31), we get

$$
\lim _{n \rightarrow \infty} F\left(\mathscr{C}\left(\varsigma_{n}, \varsigma_{n+1}\right)\right)=-\infty
$$

By $\left(F_{2}\right)$, we get

$$
\lim _{n \rightarrow \infty} \mathscr{C}\left(\varsigma_{n}, \varsigma_{n+1}\right)=0
$$

Now, by $\left(F_{3}\right)$, there is $h \in(0,1)$ so that

$$
\lim _{n \rightarrow \infty}\left[\mathscr{C}\left(\varsigma_{n}, \varsigma_{n+1}\right)\right]^{h} F\left(\mathscr{C}\left(\varsigma_{n}, \varsigma_{n+1}\right)\right)=0 .
$$

From (25), we have

$$
\begin{aligned}
{\left[\mathscr{C}\left(\varsigma_{n}, \varsigma_{n+1}\right)\right]^{h} F\left(\mathscr{C}\left(\varsigma_{n}, \varsigma_{n+1}\right)\right) } & -\left[\mathscr{C}\left(\varsigma_{n}, \varsigma_{n+1}\right)\right]^{h} F\left(\mathscr{C}\left(\varsigma_{0}, \varsigma_{n+1}\right)\right) \\
& \leq-n \tau\left[\mathscr{C}\left(\varsigma_{n}, \varsigma_{n+1}\right)\right]^{h} \leq 0 .
\end{aligned}
$$

On taking limit as $n \longrightarrow \infty$, we obtain

$$
\lim _{n \rightarrow \infty} n\left[\mathscr{C}\left(\varsigma_{n}, \varsigma_{n+1}\right)\right]^{h}=0 .
$$

Hence, $\lim _{n \rightarrow \infty} n^{1 / h} \mathscr{C}\left(\varsigma_{n}, \varsigma_{n+1}\right)=0$, and there exists $n_{1} \in \mathbb{N}$ such that $n^{1 / h} \mathscr{C}\left(\varsigma_{n}, \varsigma_{n+1}\right) \leq 1$ for all $n \geq n_{1}$. So, we have

$$
\mathscr{C}\left(\varsigma_{n}, \varsigma_{n+1}\right) \leq \frac{1}{n^{1 / h}}
$$

for all $n \geq n_{1}$. Consider the triangle inequality for $q \geq 1$ to have 


$$
\begin{aligned}
\mathscr{C}\left(\varsigma_{n}, \varsigma_{n+q}\right) \leq & p\left(\varsigma_{n}, \varsigma_{n+1}\right) \mathscr{C}\left(\varsigma_{n}, \varsigma_{n+1}\right)+p\left(\varsigma_{n+1}, \varsigma_{n+q}\right) \mathscr{C}\left(\varsigma_{n+1}, \varsigma_{n+q}\right) \\
\leq & p\left(\varsigma_{n}, \varsigma_{n+1}\right) \mathscr{C}\left(\varsigma_{n}, \varsigma_{n+1}\right)+p\left(\varsigma_{n+1}, \varsigma_{n+q}\right) p\left(\varsigma_{n+1}, \varsigma_{n+2}\right) \\
& \cdot \mathscr{C}\left(\varsigma_{n+1}, \varsigma_{n+2}\right)+p\left(\varsigma_{n+1}, \varsigma_{n+q}\right) p\left(\varsigma_{n+2}, \varsigma_{n+q}\right) \mathscr{C}\left(\varsigma_{n+2}, \varsigma_{n+q}\right) \\
\leq & p\left(\varsigma_{n}, \varsigma_{n+1}\right) \mathscr{C}\left(\varsigma_{n}, \varsigma_{n+1}\right)+p\left(\varsigma_{n+1}, \varsigma_{n+q}\right) p\left(\varsigma_{n+1}, \varsigma_{n+2}\right) \\
& \cdot \mathscr{C}\left(\varsigma_{n+1}, \varsigma_{n+2}\right)+p\left(\varsigma_{n+1}, \varsigma_{n+q}\right) p\left(\varsigma_{n+2}, \varsigma_{n+q}\right) \\
& \cdot p\left(\varsigma_{n+2}, \varsigma_{n+3}\right) \mathscr{C}\left(\varsigma_{n+2}, \varsigma_{n+3}\right)+p\left(\varsigma_{n+1}, \varsigma_{n+q}\right) \\
& \cdot p\left(\varsigma_{n+2}, \varsigma_{n+q}\right) p\left(\varsigma_{n+3}, \varsigma_{n+q}\right) \mathscr{C}\left(\varsigma_{n+3}, \varsigma_{n+q}\right) \leq \cdots \\
\leq & p\left(\varsigma_{n}, \varsigma_{n+1}\right) \mathscr{C}\left(\varsigma_{n}, \varsigma_{n+1}\right)+\sum_{i=n+1}^{n+q-2}\left(\prod_{j=n+1}^{i} p\left(\varsigma_{j}, \varsigma_{n+q}\right)\right) \\
& \cdot p\left(\varsigma_{i}, \varsigma_{i+1}\right) \mathscr{C}\left(\varsigma_{i}, \varsigma_{i+1}\right)+\prod_{i=n+1}^{n+q-1} p\left(\varsigma_{i}, \varsigma_{n+q}\right) \mathscr{C}\left(\varsigma_{n+q-1}, \varsigma_{n+q}\right) .
\end{aligned}
$$

It implies that

$$
\begin{aligned}
\mathscr{C}\left(\varsigma_{n}, \varsigma_{n+q}\right) \leq & p\left(\varsigma_{n}, \varsigma_{n+1}\right) \mathscr{C}\left(\varsigma_{n}, \varsigma_{n+1}\right)+\sum_{i=n+1}^{n+q-2}\left(\prod_{j=n+1}^{i} p\left(\varsigma_{j}, \varsigma_{n+q}\right)\right) \\
& \cdot p\left(\varsigma_{i}, \varsigma_{i+1}\right) \mathscr{C}\left(\varsigma_{i}, \varsigma_{i+1}\right)+\left(\sum_{i=n+1}^{n+q-1} p\left(\varsigma_{i}, \varsigma_{n+q}\right)\right) \\
& \cdot p\left(\varsigma_{n+q-1}, \varsigma_{n+q}\right) \mathscr{C}\left(\varsigma_{n+q-1}, \varsigma_{n+q}\right)=p\left(\varsigma_{n}, \varsigma_{n+1}\right) \\
& \cdot \mathscr{C}\left(\varsigma_{n}, \varsigma_{n+1}\right)+\sum_{i=n+1}^{n+q-1}\left(\sum_{j=n+1}^{i} p\left(\varsigma_{j}, \varsigma_{n+q}\right)\right) \\
& \cdot p\left(\varsigma_{i}, \varsigma_{i+1}\right) \mathscr{C}\left(\varsigma_{i}, \varsigma_{i+1}\right) \leq p\left(\varsigma_{n}, \varsigma_{n+1}\right) \mathscr{C}\left(\varsigma_{n}, \varsigma_{n+1}\right) \\
& +\sum_{i=n+1}^{n+q-1}\left(\prod_{j=0}^{i} p\left(\varsigma_{j}, \varsigma_{n+q}\right)\right) p\left(\varsigma_{i}, \varsigma_{i+1}\right) \mathscr{C}\left(\varsigma_{i}, \varsigma_{i+1}\right) \\
\leq & p\left(\varsigma_{n}, \varsigma_{n+1}\right) \mathscr{C}\left(\varsigma_{n}, \varsigma_{n+1}\right)+\sum_{i=n+1}^{n+q-1}\left(\prod_{j=0}^{i} p\left(\varsigma_{j}, \varsigma_{n+q}\right)\right) \\
& \cdot p\left(\varsigma_{i}, \varsigma_{i+1}\right) \frac{1}{i^{1 / k}} .
\end{aligned}
$$

Now, consider

$$
\begin{aligned}
& \sum_{i=n+1}^{n+q-1}\left(\prod_{j=0}^{i} p\left(\varsigma_{j}, \varsigma_{n+q}\right)\right) p\left(\varsigma_{i}, \varsigma_{i+1}\right) \frac{1}{n^{1 / k}} \\
& \quad=\sum_{i=n+1}^{n+q-1} \frac{1}{i^{1 / k}}\left(\prod_{j=0}^{i} p\left(\varsigma_{j}, \varsigma_{n+q}\right)\right) p\left(\varsigma_{i}, \varsigma_{i+1}\right) \\
& \leq \sum_{i=n+1}^{\infty} \frac{1}{i^{1 / k}}\left(\prod_{j=0}^{i} p\left(\varsigma_{j}, \varsigma_{n+q}\right)\right) p\left(\varsigma_{i}, \varsigma_{i+1}\right)=\sum_{i=n+1}^{\infty} U_{i} V_{i},
\end{aligned}
$$

where

$$
\begin{aligned}
& U_{i}=\frac{1}{i^{1 / k}}, \\
& V_{i}=p\left(\varsigma_{i}, \varsigma_{i+1}\right) \prod_{j=0}^{i} p\left(\varsigma_{j}, \varsigma_{n+q}\right) .
\end{aligned}
$$

Since $1 / k>0, \sum_{i=n+1}^{\infty}\left(1 / i^{1 / k}\right)$ converges and also $\left\{V_{i}\right\}_{i}$ is increasing and bounded above, thus, $\lim _{i \rightarrow \infty}\left\{V_{i}\right\}$, which is nonzero, exists. Hence, $\left\{\sum_{i=n+1}^{\infty} U_{i} V_{i}\right\}_{n}$ converges. Let us consider the partial sum

$$
S_{q}=\sum_{i=0}^{q}\left(\prod_{j=0}^{i} p\left(\varsigma_{j}, \varsigma_{n+q}\right)\right) p\left(\varsigma_{i}, \varsigma_{i+1}\right) \frac{1}{i^{1 / k}} .
$$

Now, from (39), we have

$$
\mathscr{C}\left(\varsigma_{n}, \varsigma_{n+q}\right) \leq p\left(\varsigma_{n}, \varsigma_{n+1}\right) \mathscr{C}\left(\varsigma_{n}, \varsigma_{n+1}\right)+\left(S_{n+q-1}-S_{n}\right) .
$$

By the ratio test and using the condition (26), we guarantee the existence of $\lim _{n \rightarrow \infty} S_{n}$. Hence, the real sequence $\left\{S_{n}\right\}$ is Cauchy. Now, taking the limit $n \longrightarrow+\infty$ in (43), we get

$$
\lim _{n \rightarrow \infty} \mathscr{C}\left(\varsigma_{n}, \varsigma_{n+q}\right)=0
$$

that is, $\left\{\varsigma_{n}\right\}$ is a Cauchy sequence in $(Y, \mathscr{C})$, which is complete, so $\left\{\varsigma_{n}\right\}$ converges to some $u \in Y$. We claim that $\Omega u=u$. Since $\varsigma_{n} \longrightarrow u$ as $n \longrightarrow \infty$ and $\Omega$ is continuous, we have $\Omega \varsigma_{n} \longrightarrow \Omega u$ as $n \longrightarrow \infty$. Thus, we have

$$
\mathscr{C}(u, \Omega u)=\lim _{n \rightarrow \infty} \mathscr{C}\left(\varsigma_{n+1}, \Omega u\right)=\lim _{n \rightarrow \infty} \mathscr{C}\left(\Omega \varsigma_{n}, \Omega u\right)=0,
$$

and hence, $u=\Omega u$. Thus, $u$ is a fixed point of $\Omega$. Its uniqueness is obvious.

Corollary 15. Let $(Y, \mathscr{C})$ be a complete controlled metric space, and let $\Omega: Y \longrightarrow Y$ be continuous so that

$$
\tau+F((\mathscr{C}(\Omega \varsigma, \Omega \omega)) \leq F(\mathscr{C}(\varsigma, \omega))
$$

for all $\varsigma, \omega \in Y$. For $\varsigma_{0} \in Y$, take $\varsigma_{n}=\Omega^{n} \varsigma_{0}$. Suppose that

$$
\sup _{m \geq 1} \lim _{i \rightarrow \infty} \frac{p\left(\varsigma_{i+1}, \varsigma_{i+2}\right) p\left(\varsigma_{i+1}, \varsigma_{m}\right)}{p\left(\varsigma_{i}, \varsigma_{i+1}\right)}<1 .
$$

Assume that $\lim _{n \rightarrow \infty} p\left(\varsigma_{n}, \varsigma\right)$ and $\lim _{n \rightarrow \infty} p\left(\varsigma, \varsigma_{n}\right)$ exist and are finite, for every $\varsigma \in Y$, then $\Omega$ possesses a unique fixed point.

Proof. Taking $\alpha: Y \times Y \longrightarrow[0, \infty)$ by $\alpha(\varsigma, \omega)=1$, for all $\varsigma, \omega$ $\in Y$ in Theorem 14 .

Corollary 16. Let $(Y, \mathscr{C})$ be a complete extended $b$-metric space and $\Omega: Y \longrightarrow Y$ be a continuous, $\alpha$-admissible and $(\alpha, F)$-contraction so that there is $\varsigma_{0} \in Y$ in order that $\alpha\left(\varsigma_{0}, \Omega \varsigma_{0}\right) \geq 1$. Suppose that 


$$
\sup _{m \geq 1} \lim _{i \rightarrow \infty} \frac{p\left(\varsigma_{i+1}, \varsigma_{i+2}\right) p\left(\varsigma_{i+1}, \varsigma_{m}\right)}{p\left(\varsigma_{i}, \varsigma_{i+1}\right)}<1 .
$$

If in addition, $\lim _{n \rightarrow \infty} p\left(\varsigma_{n}, \varsigma\right)$ and $\lim _{n \rightarrow \infty} p\left(\varsigma, \varsigma_{n}\right)$ exist and are finite, for every $\varsigma \in Y$, then $\Omega$ has a unique fixed point.

Corollary 17. Let $(Y, \mathscr{C})$ be a complete $b$-metric space and $\Omega: Y \longrightarrow Y$ be a continuous, $\alpha$-admissible and $(\alpha, F)$-contraction so that there is $\varsigma_{0} \in Y$ with $\alpha\left(\varsigma_{0}, \Omega \varsigma_{0}\right) \geq 1$. Then, $\Omega$ has a unique fixed point.

Proof. Taking $p: Y \times Y \longrightarrow[1, \infty)$ by $p(\varsigma, \omega)=p(\omega, \tau)$, for all $\varsigma, \omega, \tau \in Y$ in Theorem 14 .

Corollary 18. Let $(Y, \mathscr{C})$ be a complete metric space and $\Omega$ $: Y \longrightarrow Y$ be a continuous, $\alpha$-admissible and ( $\alpha, F)$-contraction so that there is $\varsigma_{0} \in Y$ in order that $\alpha\left(\varsigma_{0}, \Omega \varsigma_{0}\right) \geq 1$. Then, $\Omega$ has a unique fixed point.

Proof. Taking $p: Y \times Y \longrightarrow[1, \infty)$ by $p(\varsigma, \omega)=1$, for all $\varsigma, \omega$ $\in Y$ in Theorem 14 .

Corollary 19 (see. [11]). Let $(Y, \mathscr{C})$ be a complete metric space and $\Omega: Y \longrightarrow Y$ be an $F$-contraction. Then, $\Omega$ has a unique fixed point.

Proof. Taking $\alpha: Y \times Y \longrightarrow[0, \infty)$ by $\alpha(\varsigma, \omega)=1$, for all $\varsigma, \omega$ $\in Y$ in Corollary 18 .

\section{Data Availability}

The data used to support the findings of this study are available from the corresponding author upon request.

\section{Conflicts of Interest}

The authors declare that they have no competing interests.

\section{Authors' Contributions}

All authors contributed equally and significantly in writing this article. All authors read and approved the final manuscript.

\section{Acknowledgments}

The authors thank the Basque Government for its support of this work through Grant IT1207-19. This work has been partially supported by the Basque Government through Grant IT1207-19.

\section{References}

[1] S. Czerwik, "Contraction mappings in b-metric spaces," Acta mathematica et informatica universitatis ostraviensis, vol. 1, pp. 5-11, 1993.

[2] H. Afshari, H. Aydi, and E. Karapinar, "On generalized $\alpha-\psi$ Geraghty contractions on $b$-metric spaces," Georgian Mathematical Journal, vol. 27, no. 1, pp. 9-21, 2020.
[3] E. Ameer, H. Aydi, M. Arshad, and M. De la Sen, "Hybrid ćirić type graphic $\Upsilon, \Lambda$-contraction mappings with applications to electric circuit and fractional differential equations," Symmetry, vol. 12, no. 3, p. 467, 2020.

[4] A. Azam, N. Mehmood, J. Ahmad, and S. Radenović, "Multivalued fixed point theorems in cone $b$-metric spaces," Journal of Inequalities and Applications, vol. 2013, no. 1, 2013.

[5] H. Huang and S. Radenović, "Some fixed point results of generalized lipschitz mappings on cone $b$-metric spaces over Banach algebras," Journal of Computational Analysis \& Applications, vol. 20, pp. 566-583, 2016.

[6] E. Karapinar, S. Czerwik, and H. Aydi, " $(\alpha, \psi)-$ Meir-Keeler contraction mappings in generalized-metric spaces," Journal of Function Spaces, vol. 2018, Article ID 3264620, 4 pages, 2018.

[7] H. Qawaqneh, M. Md Noorani, W. Shatanawi, H. Aydi, and H. Alsamir, "Fixed point results for multi-valued contractions in $b$-metric spaces and an Application," Mathematics, vol. 7, no. 2, p. 132, 2019.

[8] T. Kamran, M. Samreen, and Q. UL Ain, "A generalization of $b$-metric space and some fixed point theorems," Mathematics, vol. 5, no. 2, p. 19, 2017.

[9] N. Mlaiki, H. Aydi, N. Souayah, and T. Abdeljawad, "Controlled metric type spaces and the related contraction principle," Mathematics, vol. 6, no. 10, p. 194, 2018.

[10] T. Abdeljawad, N. Mlaiki, H. Aydi, and N. Souayah, "Double controlled metric type spaces and some fixed point results," Mathematics, vol. 6, no. 12, p. 320, 2018.

[11] D. Wardowski, "Fixed points of a new type of contractive mappings in complete metric spaces," Fixed Point Theory and Applications, vol. 2012, no. 1, 2012.

[12] J. Ahmad, A. Al-Rawashdeh, and A. Azam, "New fixed point theorems for generalized $F$-contractions in complete metric spaces," Fixed Point Theory and Applications, vol. 2015, no. $1,2015$.

[13] E. Ameer, H. Aydi, M. Arshad, H. Alsamir, and M. Noorani, "Hybrid multivalued type contraction mappings in $\alpha_{K}$-complete partial $b$-metric spaces and applications," Symmetry, vol. 11, no. 1, p. 86, 2019.

[14] L. Budhia, H. Aydi, A. H. Ansari, and D. Gopal, "Some new fixed point results in rectangular metric spaces with an application to fractional-order functional differential equations," Nonlinear Analysis: Modelling and Control, vol. 25, no. 4, pp. 580-597, 2020.

[15] N. Hussain, J. Ahmad, and A. Azam, "On Suzuki-Wardowski type fixed point theorems," Journal of Nonlinear Sciences and Applications, vol. 8, no. 6, pp. 1095-1111, 2015.

[16] N. Hussain, J. Ahmad, L. Ćirić, and A. Azam, "Coincidence point theorems for generalized contractions with application to integral equations," Fixed Point Theory and Applications, vol. 2015, no. 1, 2015.

[17] Z. Ma, A. Asif, H. Aydi, S. U. Khan, and M. Arshad, "Analysis of F-contractions in function weighted metric spaces with an application," Open Mathematics, vol. 18, no. 1, pp. 582-594, 2020.

[18] M. Olgun, T. Alyildiz, O. Bicer, and I. Altun, "Fixed point results for F-contractions on space with two metrics," Filomat, vol. 31, no. 17, pp. 5421-5426, 2017.

[19] V. Parvaneh, M. R. Haddadi, and H. Aydi, "On best proximity point results for some type of mappings," Journal of Function Spaces, vol. 2020, Article ID 6298138, 6 pages, 2020. 
[20] P. Patle, D. Patel, H. Aydi, and S. Radenović, "On $H^{+}$Type multivalued contractions and applications in symmetric and probabilistic spaces," Mathematics, vol. 7, no. 2, p. 144, 2019.

[21] H. Sahin, I. Altun, and D. Turkoglu, "Two fixed point results for multivalued F-contractions on $M$-metric spaces," Revista de la Real Academia de Ciencias Exactas, Físicas y Naturales. Serie A. Matemáticas, vol. 113, no. 3, pp. 1839-1849, 2019.

[22] W. Shatanawi, E. Karapinar, H. Aydi, and A. Fulga, "Wardowski type contractions with applications on Caputo type nonlinear fractional differential equations," University Politechnica of Bucharest Scientific Bulletin-Series A-Applied, vol. 82, no. 2, pp. 157-170, 2020.

[23] S. Reich, "Fixed point of contractive functions," Bollettino dell'Unione Matematica Italiana, vol. 4, pp. 26-42, 1972.

[24] S. Reich, "Kannan's fixed point theorem," Bollettino dell'Unione Matematica Italiana, vol. 4, pp. 1-11, 1971.

[25] B. Samet, C. Vetro, and P. Vetro, "Fixed point theorems for $\alpha-\psi$-contractive type mappings," Nonlinear Analysis: Theory, Methods \& Applications, vol. 75, no. 4, pp. 2154-2165, 2012. 